\title{
Implementation of Ultrasonic Ranger Finder
}

\author{
Wen-Bin Lin ${ }^{1}$, Kao-Feng Yarn ${ }^{2}$ \\ ${ }^{1}$ Department of Electronic and Optoelectronic Application Engineering, ${ }^{2}$ Department of Aircraft Maintenance, \\ Far East University, Taiwan 744, ROC
}

Corresponding Author: Wen-Bin Lin

\begin{abstract}
In this paper, the operation style of "measuring distance" is taken as the research content of this implementation. The expected goal is to use the characteristics of the double reflection type used in the transceiver circuit to perform the function of measuring distance. Finally, the operating principle of the adopted electric circuits and the performance of the proposed device are validated by experimental results. It reveals that the operations of the proposed device coincide with the requirements.
\end{abstract}

Keywords: Ultrasound, Pulse echo method, Single frequency ranging, Verification

\section{INTRODUCTION}

Distance measurement system can be done by laser, ultrasonic, etc. They are similar to the principle of measurement, there are also pulse echo method and phase difference method. However, the choice depends on the application needs, the environment and the measurement range. Because of the relatively low cost of ultrasonic distance measurement system, and ultrasonic wave directional strong, slow energy consumption, in the medium propagation distance, and ultrasonic measurement system components are easy to obtain, for doing experiments is a very good choice. If its measurement performance can be improved, the application of ultrasonic measurement will be more extensive. The application aspects are robot, self-propelled vehicle, reverse sensing, water level measurement, camera focus and distance measurement, etc.

Based on this concept, the purpose of this paper is to study the single-frequency ultrasound ranging function to transmit ultrasound waves from an ultrasound transmitter to a certain direction. The ultrasonic wave propagates in the air and returns immediately when it meets the target on the way. The ultrasonic receiver stops timing immediately after receiving the reflected wave, and the measurement result is obtained. The propagation speed of the sound wave in the air is $340 \mathrm{~m} / \mathrm{s}$. According to the time $t$ recorded by the timer, the distance (s) between the emission point and the obstacle can be calculated, i.e. $s=340 t / 2$. This is the so-called time difference ranging algorithm (TOF). Dividing by 2 is because it is counting the round trip time and half is the distance we want. Based on this concept, this paper adopts the pulse-echo method and uses the time difference for the application of the ranging system [1-12].

The sensors used to emit sound waves above $20 \mathrm{KHz}$ or to detect the presence of sound waves above $20 \mathrm{KHz}$ are commonly referred to as ultrasonic sensors.

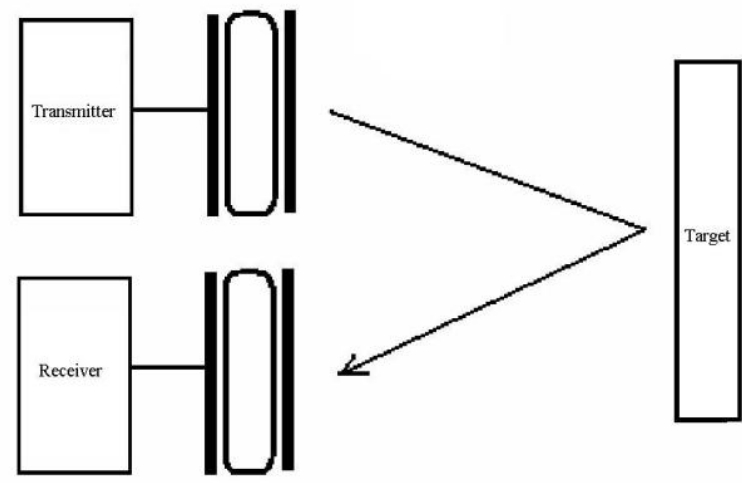

Figure 1: The block diagram for the dual-head reflective ultrasonic distance measurement system

The block diagram for the dual-head reflective ultrasonic distance measurement system is shown in Figure 1 The ultrasonic transmitter and receiver are placed in the same 
direction, the transmitter transmits the signal, and the signal hits the reflector (target) and is reflected back to be received by the receiver. The distance between the sensor and the reflected object can be calculated by using this to determine whether there is an object approaching and to know the time required for the ultrasonic wave to be emitted.

\subsection{Ultrasonic Sensor for Distance Measurement}

Ultrasonic sensors are used in a wide range of applications, such as distance measurement, flow measurement, speed detection, non-destructive inspection of material damage, and medical inspection. The main purpose of this study is to study the ultrasonic distance measurement system, so this paper only focuses on the distance measurement of the ultrasonic sensor to explain. The ultrasonic sensor for distance measurement uses the transmitter of the ultrasonic sensor to send ultrasonic waves to the receiver, and the time $(\mathrm{t})$ required to receive the response reflected from the target object to obtain the distance between the target object and the measurement source [3]. The basic measurement principle of ultrasonic distance measurement is shown in Figure 2.

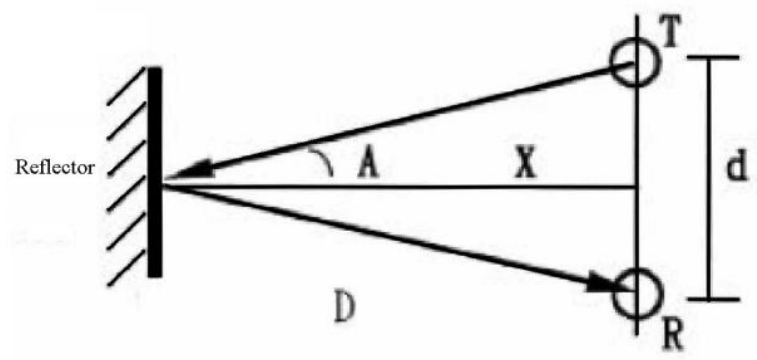

Figure 2: Basic principle of ultrasonic distance measurement

The relationship is as in equation (1).

Distance $=\mathrm{C} *$ Flight time $(\mathrm{t} / 2)$

where, $\mathrm{C}=$ Speed of sound.

The speed of the sound wave in the air is $343 \mathrm{~m} / \mathrm{s}$ at $1 \mathrm{~atm}$ and an operating temperature of $20{ }^{\circ} \mathrm{C}$. However, the speed of sound varies with temperature and the relationship is:

$$
C=331.31 \sqrt{1+\frac{T}{273.15}}
$$

T: Operating temperature $\left({ }^{\circ} \mathrm{C}\right)$

C: Sound wave speed $(\mathrm{m} / \mathrm{s})$

$$
\begin{aligned}
& X=\frac{(C \times t \times \cos A)}{2} \\
& D=C \times \frac{t}{2}
\end{aligned}
$$

$C$ : Wave speed (operating at $1 \mathrm{~atm}, 20^{\circ} \mathrm{C}$, sound wave speed $=343 \mathrm{~m} / \mathrm{s}$ ).

$t$ : Time required for transmit ultrasonic signal to receive signal

$T$ : Operating temperature $\left({ }^{\circ} \mathrm{C}\right)$

$A$ : Sound wave incidence angle

$D$ :(Sound wave actual flight distance) / 2

$X$ :Distance between sensor and target object

For distance measurement ultrasonic sensors, the measurement frequency should be increased as much as possible (for accurate echo measurement) in order to obtain a higher distance resolution when measuring distance (based on time difference). In addition, if the dual-head reflection type detection method is used, the transmitter $(T)$ and receiver $(R)$ need to be close together, otherwise there will be baseline error (refer to equations (3) and (4)). $\mathrm{X} / \mathrm{d}>10$, then $\mathrm{X}$ and $\mathrm{D}$ will be within $1 \%$ error. However, it will make the receiver resonate and affect the reception of closer reflected waves. If the transmitter and receiver are too close to each other, the waves emitted by the ultrasonic transmitter will be sent directly to the receiver of the ultrasonic sensor, causing misjudgment. If the single-head reflective detection method is used, the transmitting and receiving of the sound waves are controlled by the switching circuit, and $\mathrm{X}$ is close to $\mathrm{D}$. There is almost no baseline error. But also because of the need for transmitting and receiving switching circuit control, so it is not possible to detect the close range. It needs to be larger than $7 \mathrm{~cm}$ to be measured.

\section{ULTRASONIC PULSE ECHO METHOD DISTANCE MEASUREMENT PROCESS}

The distance is measured using not only the necessary ultrasonic sensors, but also a microprocessor that can determine and process the signal. Therefore, the signal generator is used to process the signal transmission and reception data. Figure 3 shows the system block diagram of the pulse/echo method.

As shown in Figure 3 and Figure 4, at the beginning of the measurement, a discontinuous voltage $\mathrm{V}$ can be generated by the 
circuit system or pulse generator to drive the ultrasonic transmitter probe.

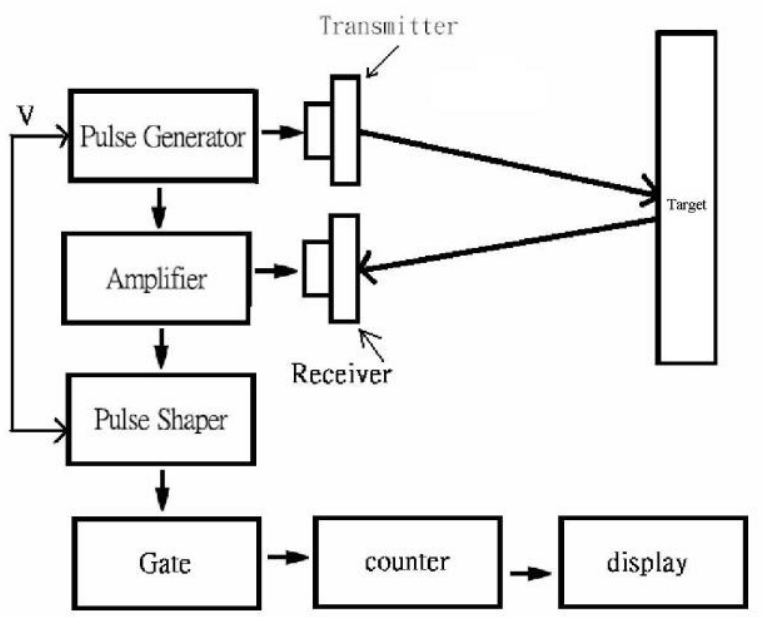

Figure 3: The system block diagram of pulse/echo method

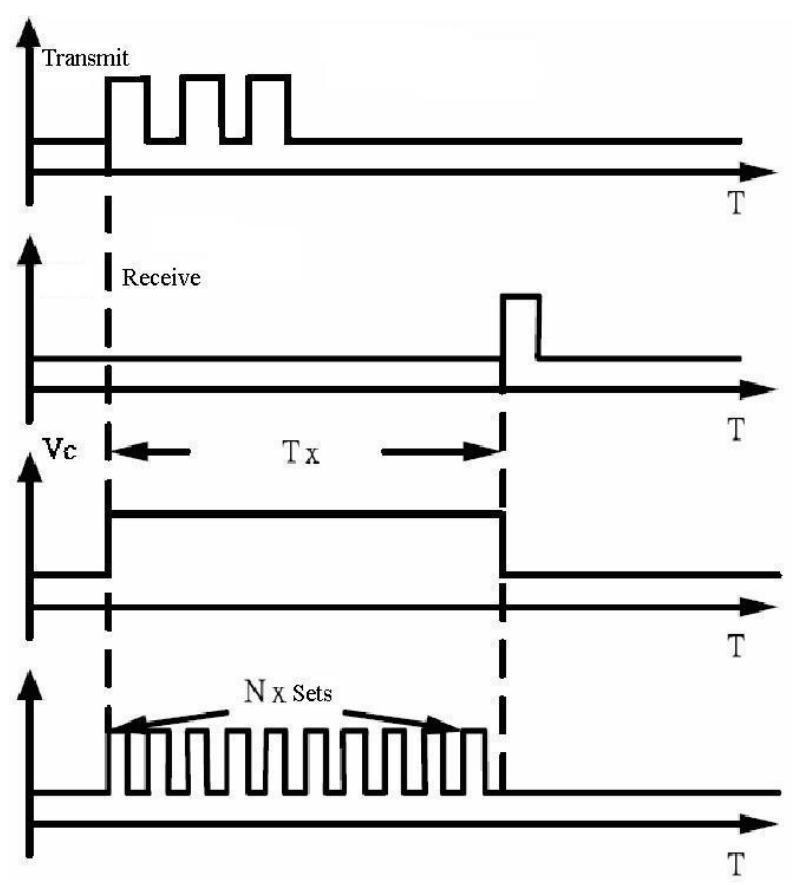

Figure 4: Time-axis description diagram of pulse/echo method [6]

The piezoelectric effect converts the electrical energy into ultrasonic pulses and advances towards the target at the speed of sound C (Velocity in air $343 \mathrm{~m} / \mathrm{s}$ ). The sound waves are bounced back from the surface of the target, and the signal is received by the ultrasonic receiver. The $\mathrm{Vc}$ signal formed by taking the transmitting and receiving leading edge waveforms respectively, and the Tx time marker signal obtained from the $\mathrm{Vc}$ signal is proportional to the distance. The $\mathrm{Vc}$ is then sent to the counter for counting and further processing by the microprocessor. This type of measurement method, it can directly convert the analog value to digital without the traditional ADC (Analog to Digital Convert) converter, so there is no need to consider the quantization error.

The following is its mathematical representation:

$$
X=C \times \frac{1}{2} T x
$$

$X$ : Distance

$C$ : Wave speed (different values depending on the dielectric)

$T_{X}$ : The time obtained from transmitting to receiving

From the above figure $\mathrm{Vc}$ to discuss the value of Tx obtained :

$T x=N_{X} \times T_{N X}$

$N_{X}$ is the number of counting

$T_{N X}$ is the time of the base frequency count

Then bring (1) into (2)

$$
\begin{aligned}
& X=C \times \frac{1}{2} T_{x}=C \times \frac{1}{2} N x \times T_{N x}=\frac{1}{2} \times \frac{C}{f_{N x}} \times N_{x} \\
& =\frac{1}{2} \times K \times N_{x}
\end{aligned}
$$

where $K=\frac{C}{f_{\mathrm{N} x}}$ is a constant

Pulse-echo method of measurement range: the use of intermittent emission, the use of greater power, can be used to measure more distant places. Pulse echo method resolution: each wave emission has a certain interval so it is difficult to find the ultrasonic wave repetition rate of vibration changes, so only the energy to measure the static or slow-moving object displacement.

\section{SYSTEM CIRCUIT DIAGRAM}

The signal generator generates a signal $\mathrm{Vpp}=20 \mathrm{~V}$, which passes through the diode to generate a positive half-period to the ultrasonic receiver transmitter. After the signal is emitted and stopped for a period of time, the return signal $\mathrm{Vpp}=5 \mathrm{mv}$, the signal is too small to the diode as an open circuit to the oscilloscope display signal waveform, as shown in Figure 5. 


\section{OSCILLOSCOPE MEASUREMENT WAVEFORM}

The distance between the transmitter and receiver is closer $(25 \mathrm{~cm})$, the signal is sent out and the receiver is reflected closer to produce, as shown in Figure 6 . The distance between the transmitter and receiver is far $(100 \mathrm{~cm})$, and the signal is transmitted and reflected far from the receiver, as shown in Figure 7.

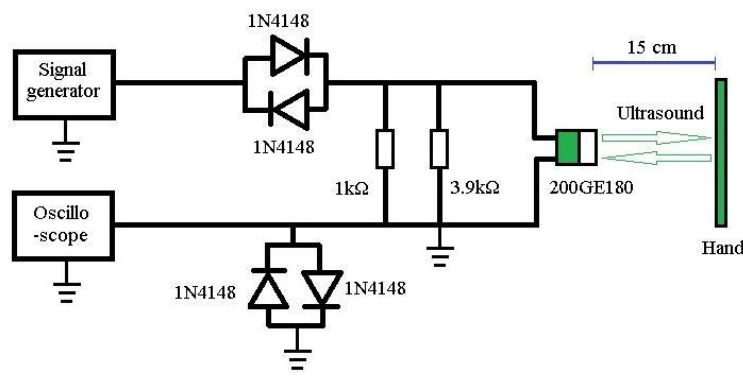

Figure 5: Circuit diagram of the ultrasonic distance measurement system

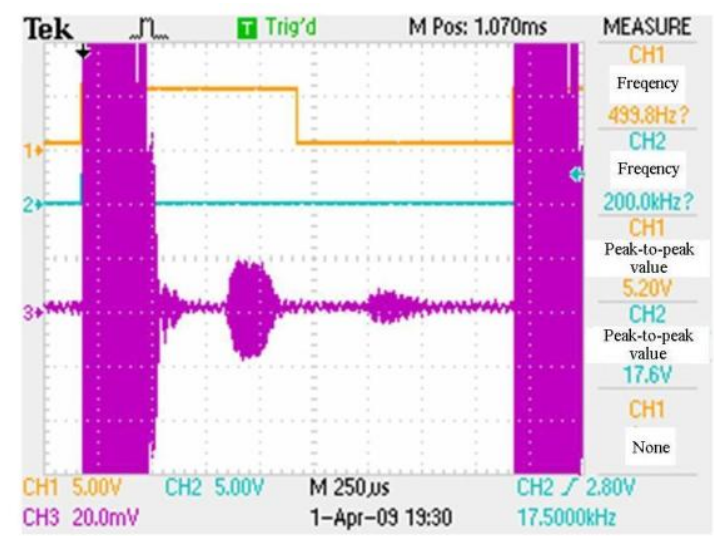

Figure 6: Ultrasonic distance measurement $(25 \mathrm{~cm})$

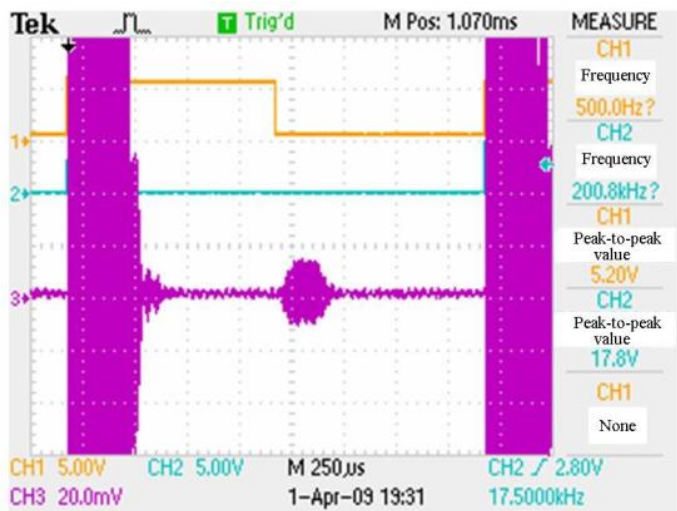

Figure 7: Ultrasonic distance measurement $(100 \mathrm{~cm})$

\section{CONCLUSION AND PRODUCTION RESULTS}

Figures 8 to 9 are photos of the hardware achievements of this paper. This paper implements a group of circuits that meet the above functions, and uses the results to verify the operation of the designed circuit and the functions that meet the requirements. It can be as an embryonic form reference for researchers engaged in related research.

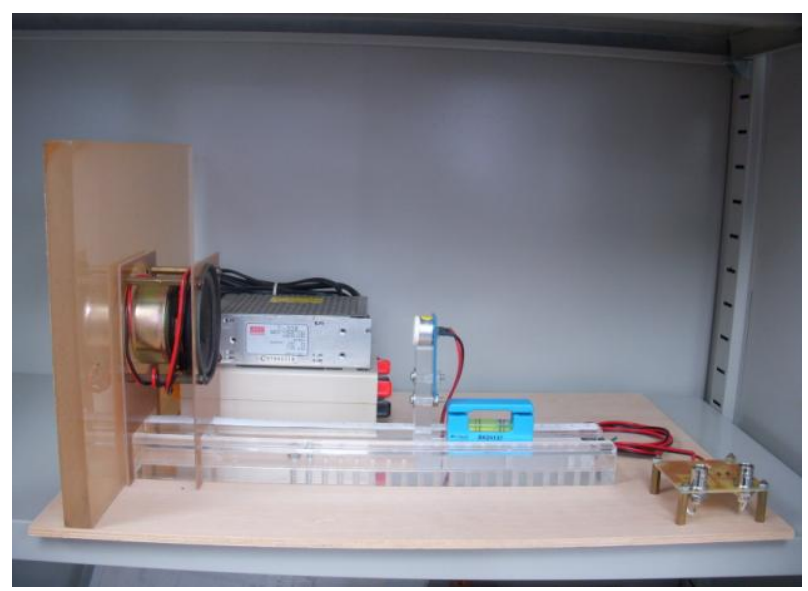

Figure 8: Side view of finished product

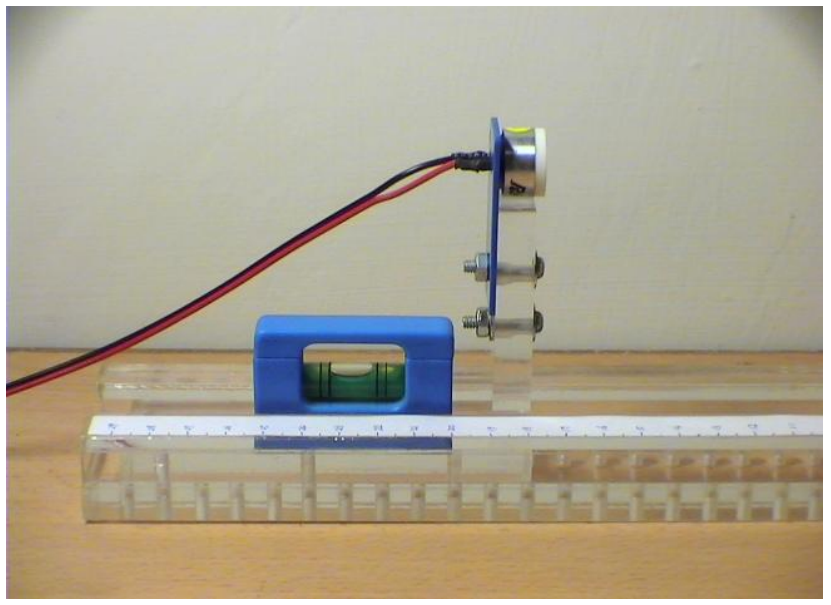

Figure 9: Ultrasonic Receiver and Transmitter

\section{ACKNOWLEDGMENTS}

The authors would like to acknowledge Prof. Long $\mathrm{Wu}$ who constructed much of the hardware for the experimental system.

\section{Conflict of Interest: None}

\section{Source of Funding: None}

\section{REFERENCES}

1. M.K. Huang, , A Compendium of Ultrasonic Inspection Practices for Industrial Use, China Shipbuilding Corporation, pp. 1 2, June 1997. 
2. R. H. Chen, Sensors, Chuan-Hwa Books Co., Ltd., pp. 479-484, October, 1994.

3. Y. Han and H. Hahn, "Localization and Classification of Target Surfaces Using Two Pairs of Ultrasonic Sensors," Elsevier Science in Robotics and Autonomous Systems, Vol.1, pp.31-41, 2000.

4. Yao-Kuang Chung, Piezoelectric MicroUltrasonic Sensor, Master thesis of National Taiwan University, June, 2001.

5. S.C Liao, The Simulation of the Resonance Effect on Blood Pressure and Flow, Master thesis of National Taiwan University, 1992.

6. H.Y. Lin, Pulse/Echo Ultrasonic Organ Micro-Resonance Measurement System, Master thesis of National Cheng Kung University, June, 2002.

7. C.F. Chang, Research and Development of Phase Difference Ranging System for Heterogeneous Wave Velocity Media, Master thesis of Central University, July, 2003.
8. N. J. Chen, Introduction and Application of Combinatorial Languages, Jurin Book Publishing Co., Ltd., 2003.

9. R. L. Boylestad, and L. Nashelsky, Electronic Devices and Circuit Theory, Ninth Ed., Pearson Education, Inc, 2005.

10. Y. Ando, and S. Yuta, "Following a Wall by an Autonomous Mobile Root with a Sonar-Ring," IEEE International Conference on Robotics and Automation, Vol.4, pp.2599-2606, 1995.

11. Y.P Hwang, Application of Contaminants Sensing and Capturing Techniques to Range Hood during Home Cooking, Master thesis of National Cheng Kung University, 2000.

12. Stefan Kocis, "Ultrasonic Measurements and Technologies", PP.64-76, Chapman \& Hall, UK, 1996.

How to cite this article: Wen-Bin Lin, Kao-Feng Yarn. Implementation of Ultrasonic Ranger Finder. International Journal of Research and Review. 2021; 8(4): 386-390. DOI: https:// doi.org/10.52403/ijrr.20210445 